\title{
Real-time time correlated photon counters for photon number resolving detectors
}

Sadik, Mala, Ai, Xiao, Lu, Yang, Nock, Richard

Mala Sadik, Xiao Ai, Yang Lu, Richard Nock, "Real-time time correlated photon counters for photon number resolving detectors," Proc. SPIE 11354, Optical Sensing and Detection VI, 1135406 (1 April 2020); doi: $10.1117 / 12.2555995$

SPIE. Event: SPIE Photonics Europe, 2020, Online Only 


\title{
Real-time time correlated photon counters for photon number resolving detectors
}

\author{
Mala Sadik, Xiao Ai, Yang Lu and Richard Nock \\ School of Engineering \& Applied Science, Aston University \\ Birmingham, UK
}

\begin{abstract}
In this paper, a novel Field Programmable Gate Array (FPGA) based instrument for Time Correlated Photon Counting (TCPC) is reported which takes advantage of the photon number resolving ability of newer single photon detectors technologies, such as silicon photomultipliers ( $\mathrm{SiPM}$ ). Utilizing the received photon number in the formation of the TCPC histogram will enable faster measurements in comparison to Time Correlated Single Photon Counting (TCSPC) as less information is being discarded per excitation pulse. The concept of (TCSPC) and the differences with TCPC are introduced, as the well as the advantages of using TCPC when using a number resolving photodetector such as a SiPM rather than a traditional Single Photon Avalanche Diode (SPAD). The design and performance of the system is reported. An 8-stop TCPC system is presented along with a hardware solution to handle time correlations in real-time on-chip rather than in post processing. A system accuracy of 42ps RMS has been demonstrated.
\end{abstract}

Keywords: Time Correlated Photon Counting, time-to-digital converter, Silicon Photomultiplier, LIDAR.

\section{INTRODUCTION}

This paper reports on the first real-time time correlated photon counting (TCPC) system and presents an instrument which can be used as an alternative to time correlated single photon counting (TCSPC) systems for photon number resolving detectors. Traditional TCSPC systems using single photon avalanche diode (SPAD) detectors typically keep the received photon number at 0.1 photons per excitation pulse or less to ensure that the probability distribution of received photons can be accurately recorded [1], which increases the integration time required to perform a measurement. However, photon number resolving detectors such as silicon photomultipliers (SiPM) offer time of arrival and photon number resolving abilities [2,3]. To date, the vast majority of systems using SiPM do not make use of the photon number resolving capability of such detectors. The advantage of TCPC over TCSPC is that the photon number information contained in every photon detection event can be used to build up a histogram faster than in a traditional TCSPC setup, allowing for shorter integration times under the same experimental conditions. To achieve the first real-time TCPC system, the number resolving capabilities of a silicon photomultiplier have been studied and a custom instrument has been developed.

\section{BACKGROUND}

Time correlated single photon counting (TCSPC) is a measurement technique used to record optical waveforms with high accuracy by using repetitive measurements $[1,4]$. It is commonly used in applications such as light detection and ranging (LiDAR) [5], the characterization of light sources, and fluorescence-lifetime imaging microscopy (FLIM) [4]. TCSPC instruments typically work by taking numerous time difference measurements between the pulse used to interrogate the system being measured and the returned photon, if present, as shown in Figure 1. These time differences are typically measured by a time-to-digital converter (TDC) over an integration time, with these time differences being shown as a histogram which represents the shape of the received optical waveform.

Optical Sensing and Detection VI, edited by Francis Berghmans, Anna G. Mignani, Proc. of SPIE Vol. 11354, 1135406 - (C) 2020 SPIE · CCC code: 0277-786X/20/\$21 - doi: 10.1117/12.2555995 


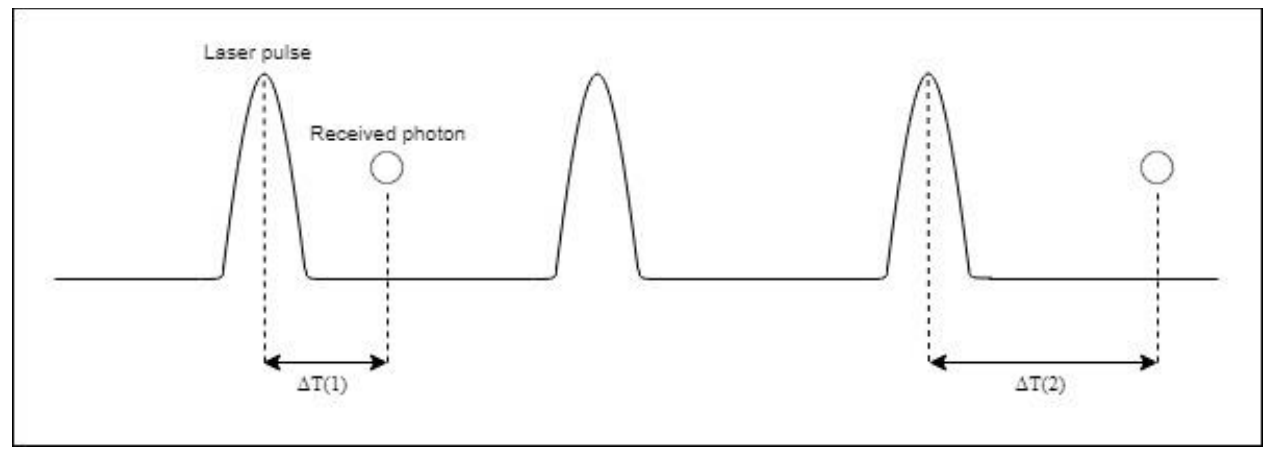

Figure 1: Measurements performed when using TCSPC

$$
\Delta T(i)=T_{S T O P}-T_{S T A R T}(i)
$$

A single time difference between the START tag $i$ and the subsequent STOP tag is calculated in (1). This method can be expanded on to become a single start - multi stop method. In this method multiple subsequent STOP tags from the START tag are used to calculate multiple time differences for the same excitation period. This is calculated using the formula:

$$
\Delta T(i, j)=T_{S T O P}(j)-T_{S T A R T}(i)
$$

Where $j$ is an integer representing the STOP tag being used, and $i$ represents the START tag.

Silicon photomultipliers (SiPM) are a new type of solid-state photodetectors. They are made up of an array of SPAD detectors whose anodes and cathodes are connected to each other. They require lower operating voltages as compared to SPADs as well as having a lower cost [4]. Because of their unique composition they have the ability to resolve the number of incident photons. When a single photon is detected a single SPAD in the array will produce a current spike on the output. If more than one photon is detected across the array, multiple SPADs in the array fire and this produces a current spike on the output whose amplitude is proportional to the number of photons detected in an event. While SiPMs have been used as alternatives to SPADs in LiDAR applications as seen in $[6,7]$, they have only been used with a single photon detection threshold. This discards the amplitude information presented by the detector and adjusting these systems to measure the number of photons per event will result in an increase in the number of photons gathered per excitation pulse. TCPC has been shown in [2] but here post processing of oscilloscope data is used to get the final measurement, which limits the use in real-time applications. 


\section{METHODOLOGY}

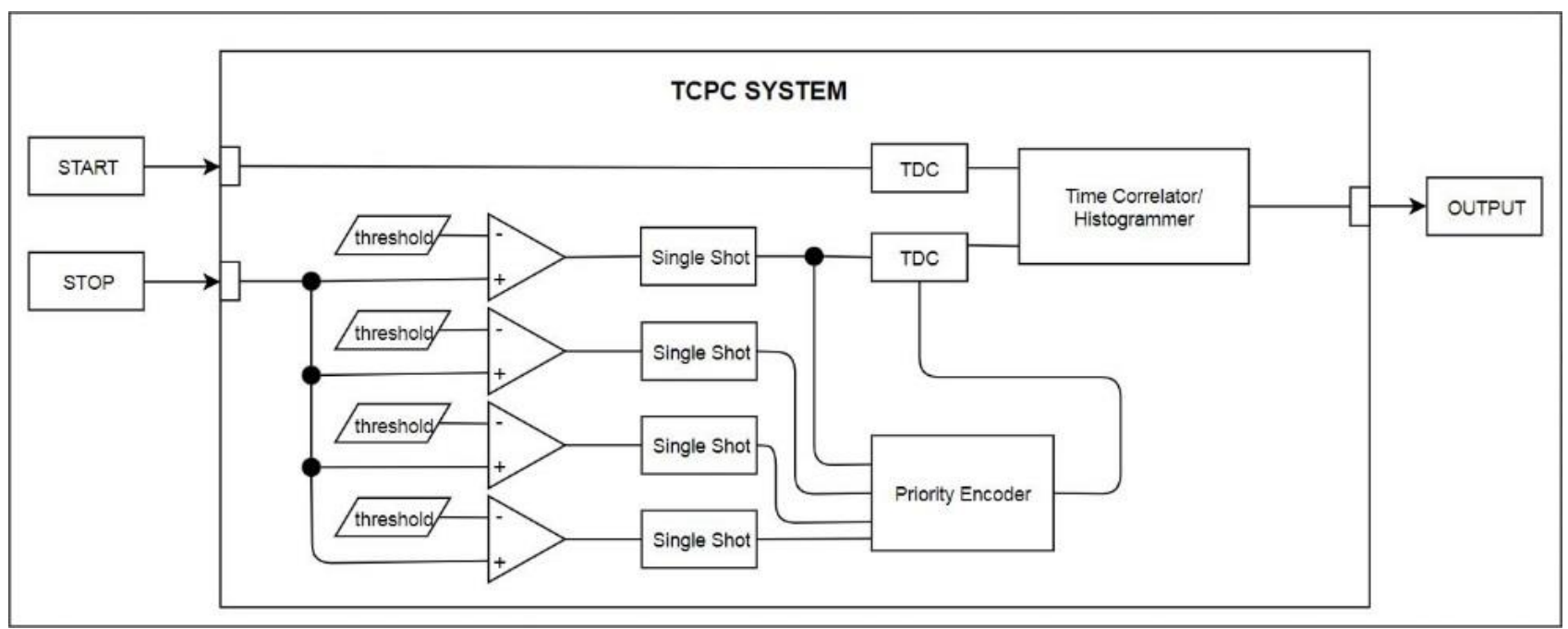

Figure 2: Block diagram of TCPC system

To achieve this, a system has been developed and a block diagram of the system is shown in Figure 2. The START signal is routed directly into a TDC which does not measure pulse amplitude. The STOP input is routed to four discriminators, each with a user configurable threshold voltage such that the amplitude of the pulse can be quantized. After this, the output of each discriminator is used to fire a single shot circuit, which comprises of a D-type flip-flop which is clocked by the output of the discriminators with the input being set to VCC. The discriminators being used are constant level discriminators (CLD) where the threshold voltage is held at a fixed value. These discriminators are best suited for signals where the pulse amplitude and rise time perturbations are minimal, which is not ideal but performs reasonably well in experimental tests. The alternative is to use constant fraction discriminators (CFD) where the threshold is dynamically set to a function of the pulse amplitude. $[4,8]$

The rationale behind the use of the single shot circuit is two-fold. Firstly, the detector pulse is short [9] (typically much less than the 8ns clock period of the TDC) and therefore, the system is likely to miss events as the TDC looks for a 0-to-1 change of state across clock cycles on the input (rising edge) to perform a measurement. Secondly, the output of all singleshot circuits needs to be synchronized to the system clock such the amplitude information can be reliably measured with no implications of subsequent registers going metastable. A pair of D type flip flops are used to reset the single shot circuits in the next 2 clock cycles and the design is pipelined to minimize dead time. Figure 3 shows a block diagram of the single shot circuit.

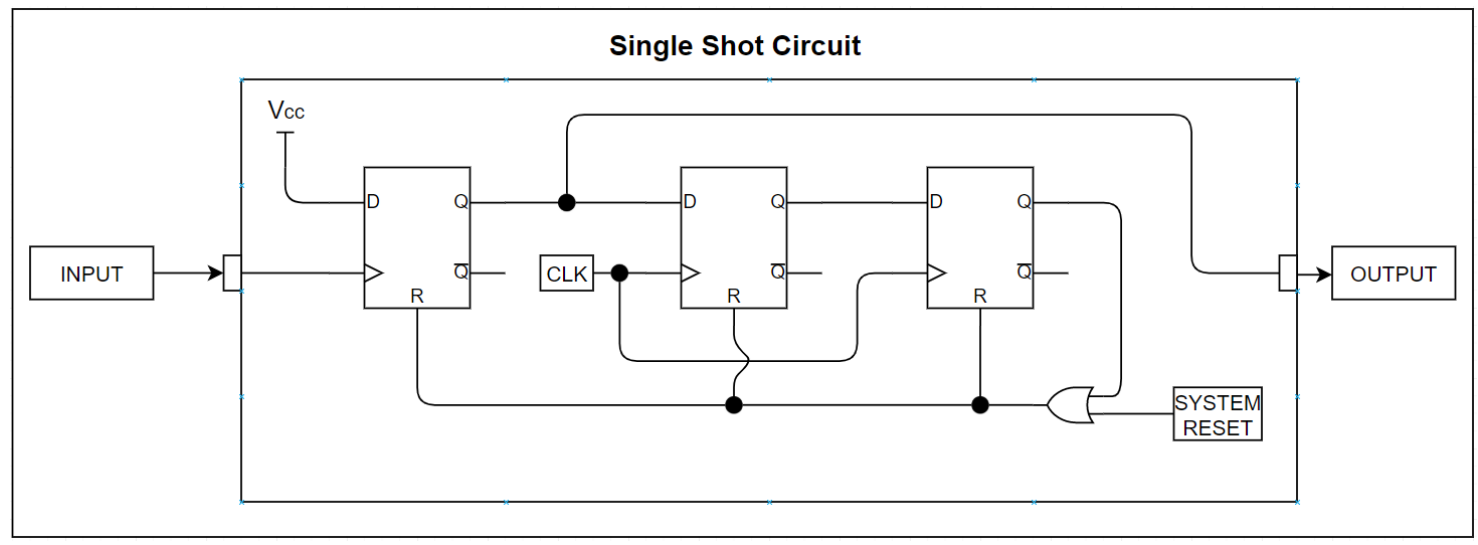

Figure 3: Single Shot Circuit Block Diagram 
A priority encoder is utilized to convert the thermometer code generated by the comparators and single shot circuits into a 2-bit vector. The output of the least significant single shot circuit (for 1 photon events) is copied to the TDC as well as the priority encoder, this copy of the signal is used to determine the time difference between the two channels. As the flip-flop is clocked from the detector input, the time information contained in this event is preserved. The TDC then passes both the time difference and the amplitude vector to the histogrammer module. The histogrammer then calculates the time correlation and uses the amplitude vector information to determine the number of counts to add to the bin located at the time difference $\Delta \mathrm{T}$. Once the integration period has completed, the histogram is transferred to the PC for viewing and analysis.

\section{RESULTS \& DISCUSSION}

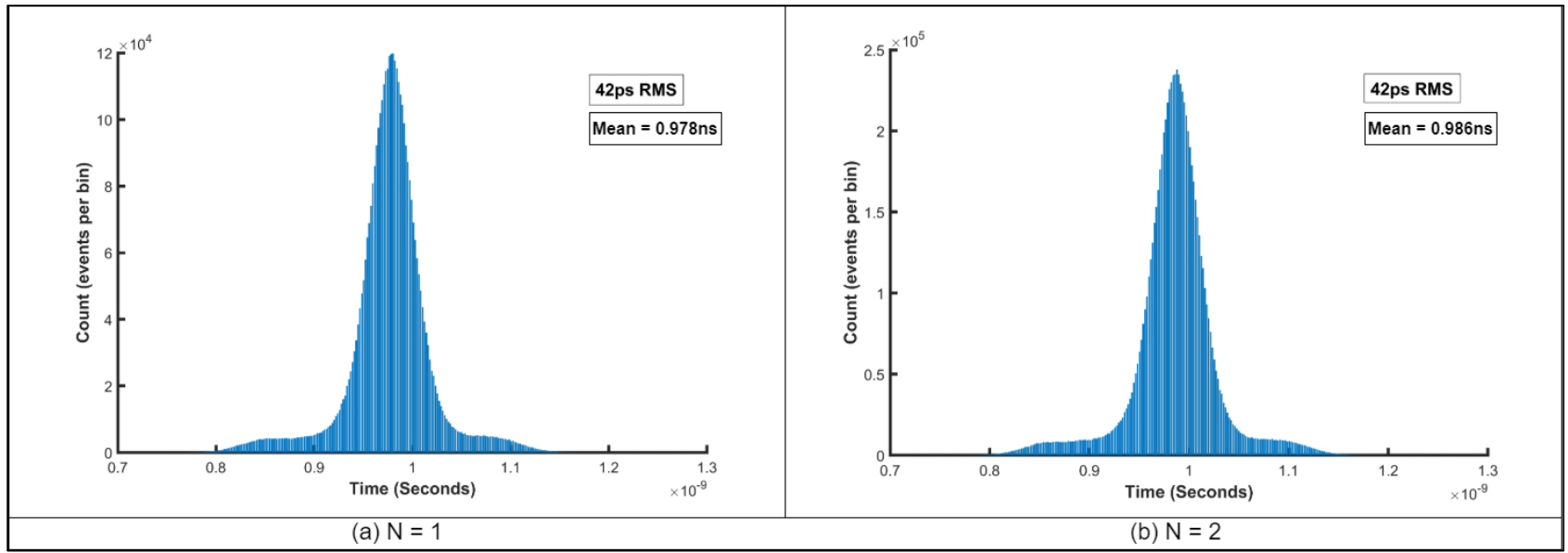

Figure 4: (a) TCSPC measurement taken using custom instrument simulating single photon events (b) TCPC measurement simulating 2 photon events

Figure 4 and 5 show the various measurements taken using the instrument while presenting the same input on the START and STOP using a HP8082A pulse generator and a Mini-Circuits ZFRSC-42-S+ $50 \Omega$ splitter. The different measurements each represent a different number of comparators being activated on the STOP input. There are 4 graphs to show the instrument works as it is supposed to and scales according to the number of comparators being triggered, which is depicted by $\mathrm{N}$. The instruments accuracy was measured to be 42ps RMS.

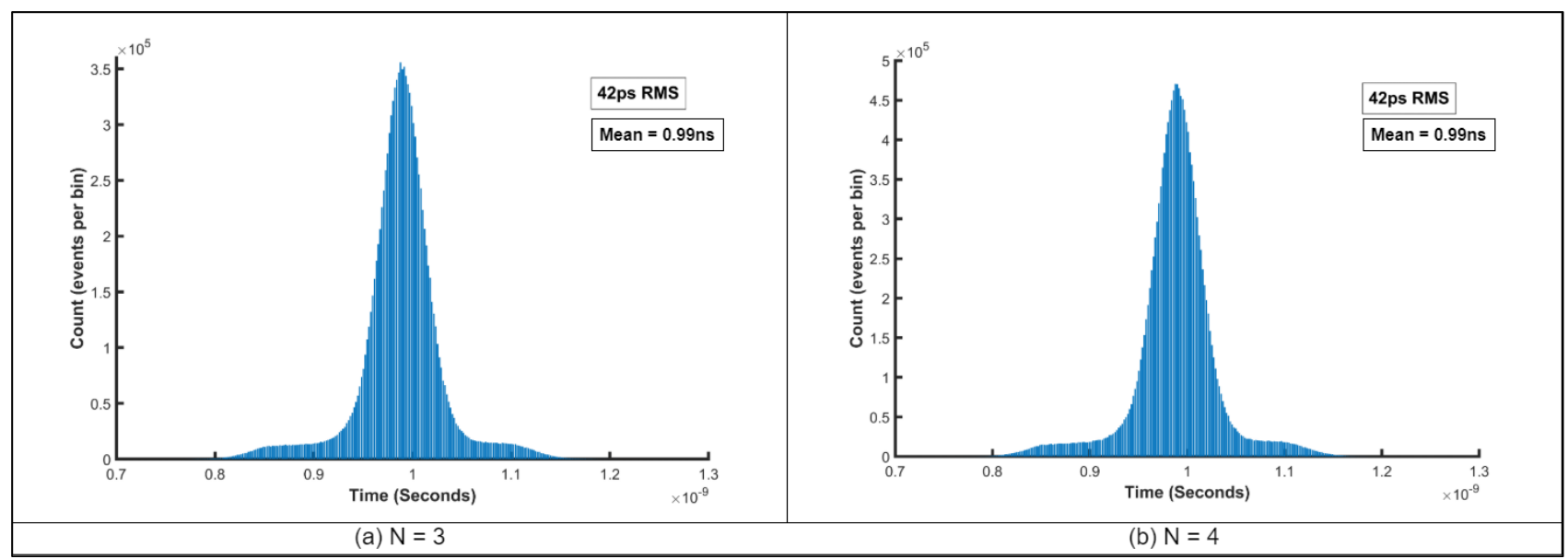

Figure 5: (a) TCPC measurement simulating 3 photon events (b) TCPC measurement simulating 4 photon events 
Time walk can be observed on the measurements seen in Figures 4 and 5 which is due to the constant level thresholding used on the comparators. This can be seen with the varying mean times for each of the graphs. Time walk mitigation techniques and CFDs will be investigated in future work to minimize input amplitude dependent time walk.

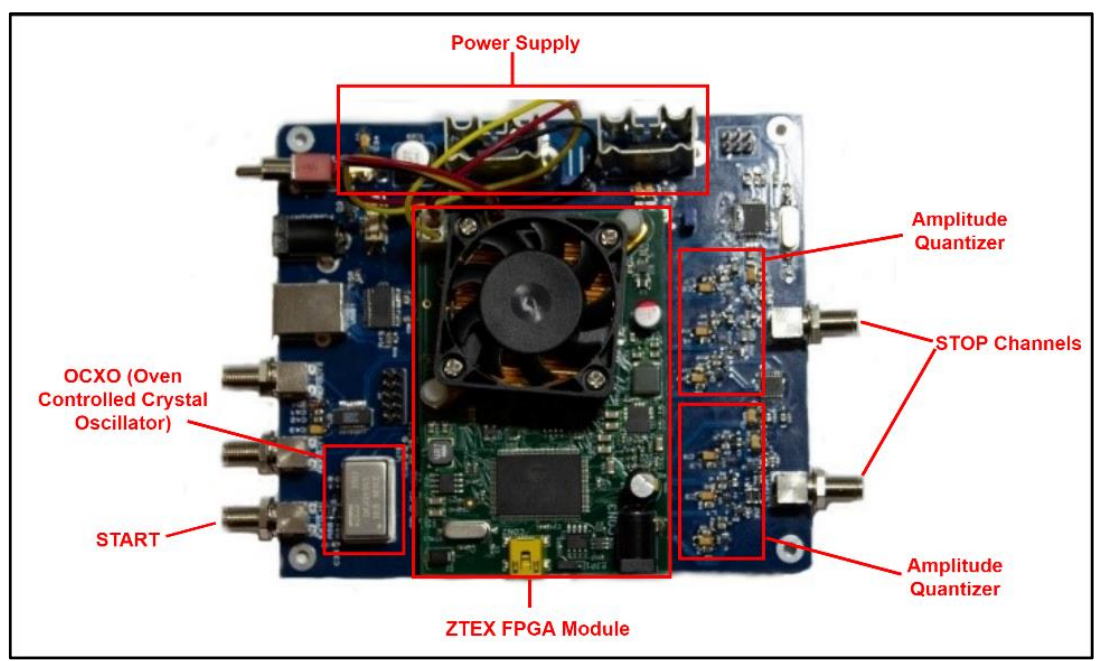

Figure 6: Picture of FPGA based TCPC system

Figure 6 shows the single start -8 stop hardware developed and used in all tests. It should be noted that the instrument can support 2 separate STOP channels each with their own amplitude quantizer on the input. The FPGA module is run using a $10 \mathrm{MHz}$ Oven Controlled Crystal Oscillator (OCXO) to provide a stable clock for the TDC. The clock output of the OCXO is put into the FPGA where a delay locked loop (DLL) [10] is used to generate the system's clock. The clock output from the DLL exhibits considerable jitter, hence, a jitter attenuator is used to minimalize the noise on the clock fed to the TDC.

The dead time for the system is limited by the single shot circuit reset time and was measured to be $18 \mathrm{~ns}$. This was measured by presenting an input on one of the single shot circuits using a HP8082A pulse generator and outputting the single shot circuit output to an oscilloscope to measure the maximum time for the circuit to reset.

The number of total counts per histogram increases in proportion to the number of photons received per START-STOP event measured as seen in Figure 7. Note that the number of photons per event was simulated via changing the threshold voltages of the discriminators accordingly. These initial results were what was expected of the system.

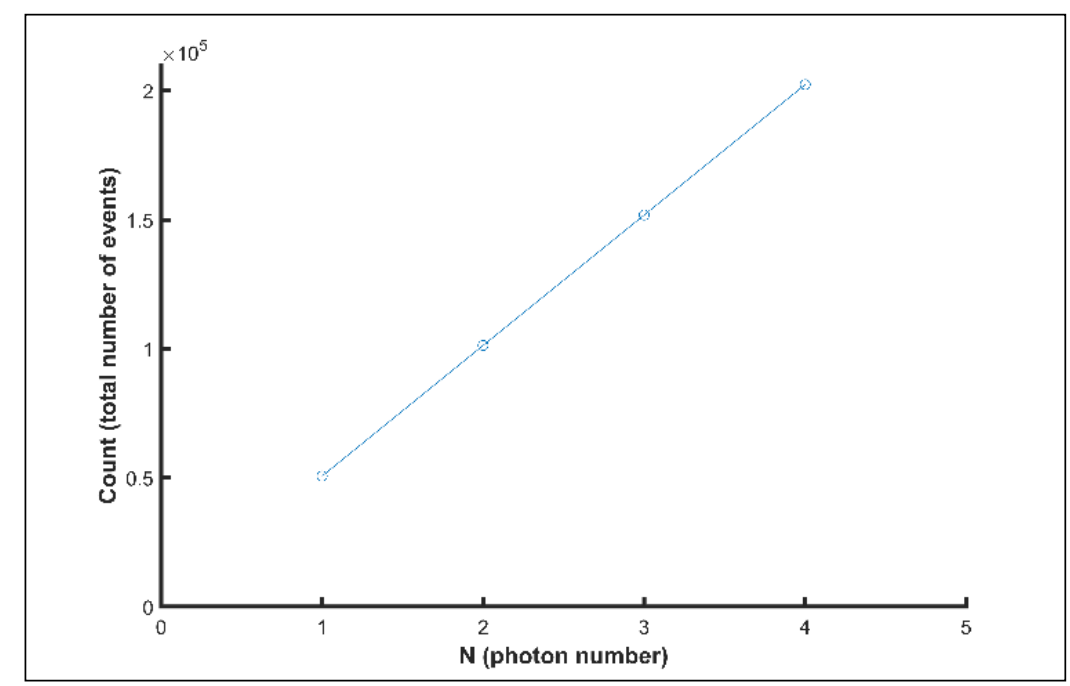

Figure 7: Plot of Total counts in histogram vs photon number 
Figure 8 shows two measurements taken using the SiPM (SensL MicroRB-10035) and the instrument developed. The same experimental setup was used in both measurements and they were both run for the same integration time. The SiPM was pulsed using the HP8082A pulse generator. A $50 \Omega$ splitter was used such that an LED and the START input of the TCPC device could be driven at the same time. The LED was coupled to the SiPM using a multimode fiber and a variable neutral density filter, such that the count rate could be adjusted optically. The HP8082A was set to output pulses with a pulse width of $5 \mathrm{~ns}$ at a frequency of approximately $20 \mathrm{MHz}$. The output of a SiPM goes through 2 stages of RF amplification from a pair of Mini-Circuits ZFL-1000LN+ amplifiers for a total gain of 40dB. Because the SiPM utilized produces negative going pulses $[9,11]$, a bias tee is used to add a DC offset to the output after the amplifiers.

Figure 8 (a) shows a TCSPC measurement taken using the experimental setup with only the single photon comparator set to a threshold for single photon events while the other comparators were turned off. In Figure 8 (b) the remaining 3 comparators are configured with their thresholds set to the voltage level of the photon number event that they respectively represent. The measurement shown is for a single dataset as there were some issues with the bias tee reference voltage drifting. This will be investigated further and corrected for in future work.

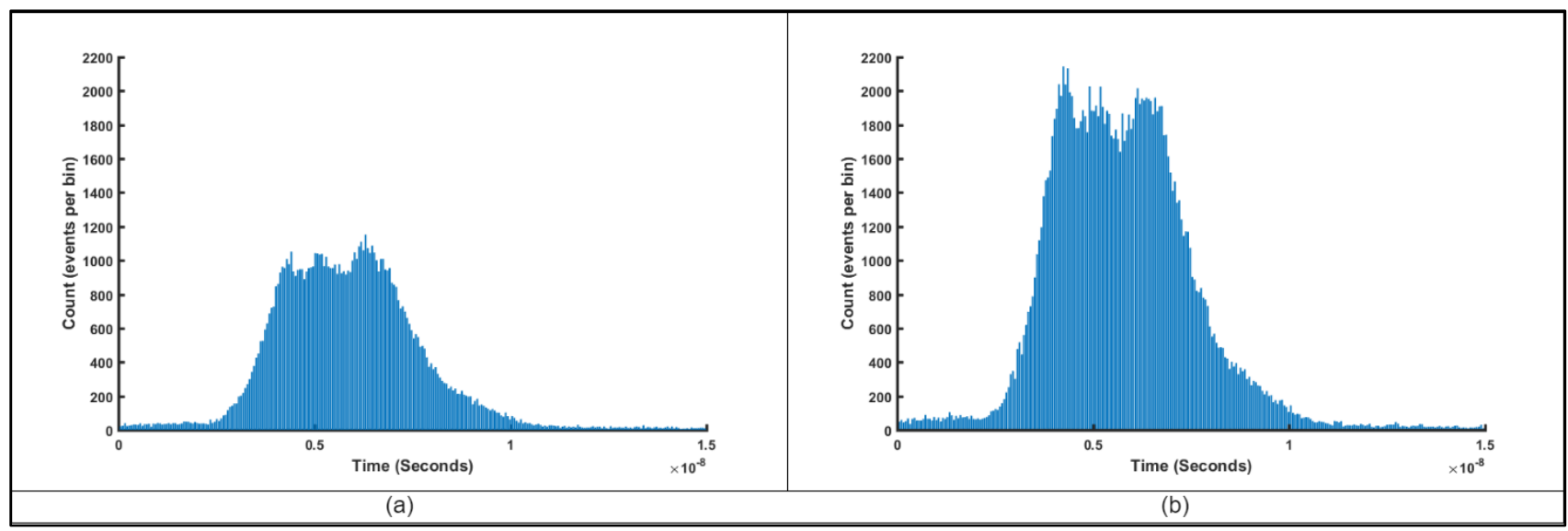

Figure 8: (a) TCSPC measurement using SiPM (b) TCPC measurement using SiPM

\section{CONCLUSION}

An instrument capable of real-time TCPC has been presented which takes advantage of the photon number resolving capabilities of silicon photomultipliers. This is achieved by using an amplitude quantizer on the detector input, which provides the amplitude vector information that is then used to determine how many counts to add to a bin in every event. The systems performance has been tested with initial results showing the instrument can extract the amplitude information in an event and use it when taking TCPC measurements. The system is capable of a timing accuracy of 42ps RMS and input quantizer determines the amount of which is added to a particular histogram bin.

Preliminary results from testing with a real SiPM show that there is a benefit to TCPC over TCSPC when using a photon number resolving detector. Future work will cover an evaluation of the benefits of TCPC over TCSPC. It is envisaged that TCPC will enable certain measurements to be taken at faster rates. 


\section{REFERENCES}

[1] W. Becker, [The bh TCSPC Handbook], 7th ed. Becker \& Hickl GmbH, (2017).

[2] Baicheng Li, Quanlong Miao, Shenyuan Wang, Debin Hui, Tianqi Zhao, Kun Liang, Ru Yang, Dejun Han, "Time-Correlated Photon Counting (TCPC) technique based on a photon-number-resolving photodetector," Proc. SPIE 9858, Advanced Photon Counting Techniques X, 98580L (5 May 2016).

[3] An Introduction to the Silicon Photomultiplier Technical Note, SensL, February 2011 <https://www.sensl.com/downloads/ds/DS-MicroRBseries.pdf> (4 March 2020).

[4] M. Wahl, "The Principle of Time-Correlated Single Photon Counting," PicoQuant, 2014, <https://www.picoquant.com/images/uploads/page/files/7253/technote_tcspc.pdf> (31 March 2019).

[5] F. Arvani, T. C. Carusone and E. S. Rogers, "TDC Sharing in SPAD-Based Direct Time-of-Flight 3D Imaging Applications," 2019 IEEE International Symposium on Circuits and Systems (ISCAS), Sapporo, Japan, (2019).

[6] G. Adamo, L. Mistretta, P. Livreri and A. Busacca, "A LiDAR Prototype with Silicon Photomultiplier and MEMS Mirrors," 2018 IEEE 4th International Forum on Research and Technology for Society and Industry (RTSI), Palermo, (2018).

[7] G. Barile, A. Leoni and M. Muttillo, "SiPM based Laser Imaging Detection and Ranging VCII Interface," 14th International Conference on Advanced Technologies, Systems and Services in Telecommunications (TELSIKS), Nis, Serbia, 2019, pp. 372-375. (2019)

[8] H. Lim "Constant Fraction Discriminator Involving Automatic Gain Control to Reduce Time Walk" IEEE Transactions on Nuclear Science, vol. 61, no. 4, (August 2014).

[9] RB-Series Datasheet SensL's Second Generation of Red-Enhanced Silicon Photomultipliers, SensL, June 2018 $<$ https://www.sensl.com/downloads/ds/DS-MicroRBseries.pdf> (4 March 2020)

[10] G. Roberts, M. Ali-Bakhshian "A Brief Introduction to Time-to-Digital and Digital-to-Time Converters," IEEE Transactions on Circuits and Systems II: Express Briefs, vol. 57, no. 3, (March 2010).

[11]Biasing \& Readout Technical Note, SensL, March 2018 <https://www.sensl.com/downloads/ds/DSMicroRBseries.pdf> (4 March 2020). 